\title{
Unraveling the stereochemically rich 2-pyridone [4+4] photodimer
}

\author{
Peiling Chen, Patrick J. Carroll, ${ }^{a}$ and Scott McN. Sieburth* \\ Department of Chemistry, Temple University, Philadelphia, PA 19122 USA \\ ${ }^{a}$ Department of Chemistry, University of Pennsylvania, Philadelphia, PA 19104 USA \\ E-mail: scott.sieburth@temple.edu
}

Dedicated to Cynthia and Bruce Maryanoff

\begin{abstract}
Photodimerization of 2-pyridones produces primarily a trans tricyclic product with a center of symmetry. Treatment of this product with chlorine leads to an amide nitrogen migration and formation of a chiral product with six stereogenic centers. We have explored the chemistry of this readily available product with the goal of producing a stereo-defined acyclic product and report here the preparation and crystal structure of a ten-carbon product rich with stereochemistry. Opening of two bridging lactams has proven to be difficult.
\end{abstract}

Keywords: Photochemistry, stereochemistry, rearrangements

\section{Introduction}

Among the [4+4] photocycloadditions, ${ }^{1}$ the photodimerization of 2-pyridones, $\mathbf{1}$, is one of the most efficient. Nevertheless, explorations of this reaction are limited and the application of this reaction for the synthesis of new structures has seen even less attention. ${ }^{2}$ This is despite the fact that four asymmetric centers are formed in this reaction from a simple precursor, with a wide tolerance for substitution, and a terpene-like ten-carbon product. ${ }^{3}$ The 2-pyridone photocycloaddition has a strong regioselectivity for head-to-tail addition, and a less pronounced stereoselectivity, favoring trans- 2 over cis- 4 (Scheme 1). The cis products are thermally unstable, undergoing Cope rearrangement under mild conditions, sometimes even at ambient temperature. ${ }^{3,4}$

The tricyclic structure 2 places the alkenes in close proximity to the amides, and in structure $\mathbf{4}$ the alkenes are in close proximity to each other. We have found that treatment of $\mathbf{2}$ and $\mathbf{4}$ with chlorine leads to products 3 and $\mathbf{5}$, respectively (Scheme) 1., ${ }^{5,6}$ The migration of the amide nitrogen during chlorination of trans- 2 occurs in essentially quantitative yield, and transforms achiral 2, which has a center of symmetry, into chiral 3 while increasing the number of asymmetric centers from four to six. 

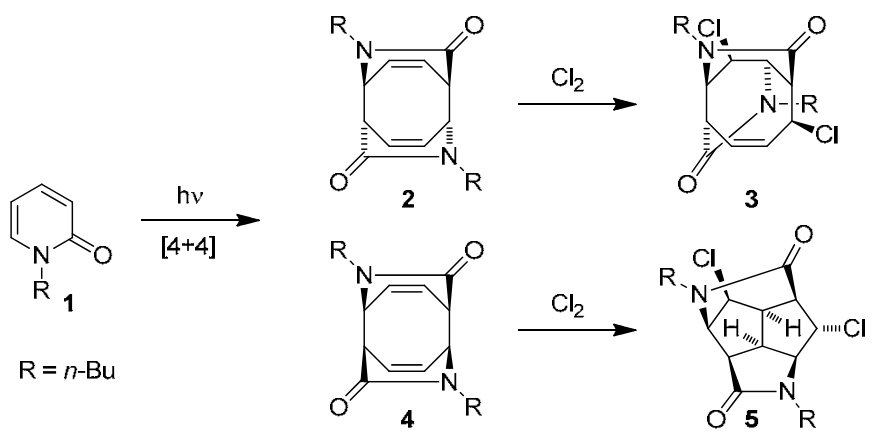

Scheme 1. Photodimerization of 2-pyridone and the major products resulting from chlorination of the trans- and cis- adducts, 2 and $\mathbf{4}$, respectively. ${ }^{5,6}$

That this simple two-step sequence generates a densely functionalized chiral structure was inspiration to further explore its potential to generate stereochemically rich asymmetric sequences by cleavage of the alkene, Scheme 2. A further intriguing feature of these molecules was the observation that the allylic chloride in $\mathbf{3}$ was readily displaced by nucleophiles. Treatment of this dichloride with sodium ethoxide leads to a quantitative displacement of the allylic chloride with retention of stereochemistry, yielding $\mathbf{6}$, Scheme $2 .^{7}$

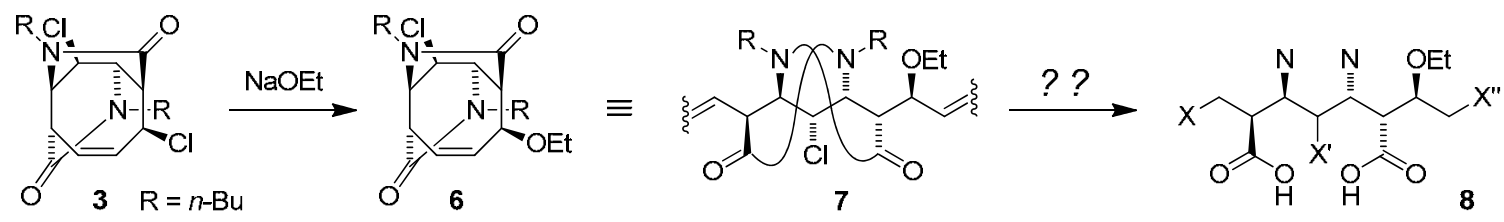

Scheme 2. Unique reactivity of the dichloride and the potential to generate stereochemically rich structures.

The mechanisms for these reactions are described in Schemes 3 and 4. Considering the three dimensional structure of $\mathbf{2}$, chlorination of an alkene can only occur from one face. For the same reason, the steric hindrance of by the proximal amide, opening of the chloronium ion $\mathbf{9}$ by chloride cannot occur. The ion is therefore opened by the amide nitrogen that lies directly underneath. The resulting quaternized nitrogen intermediate $\mathbf{1 0}$ is then dealkylated in a process reminiscent of the von Braun reaction. ${ }^{8,9}$ Of the three possible dealkylation paths for $\mathbf{1 0}$, only one is observed, yielding 3 . We were initially of the opinion that the allylic nature of this scissile bond was responsible for the site selectivity of the $\mathrm{C}-\mathrm{N}$ bond cleavage. When the crystal structure of $\mathbf{3}$ was inspected, the allylic $\mathrm{C}-\mathrm{Cl}$ bond was found to be orthogonal to the alkene $\pi$ bond, and modeling suggested that the corresponding $\mathrm{C}-\mathrm{N}$ bond of $\mathbf{1 0}$ was also poorly aligned with the $\pi$ orbitals. When displacement of the chloride by ethoxide was tested without the alkene, no change in the site of reaction was observed, suggesting that the observed allylic reactivity of $\mathbf{3}$ was primarily a result of the steric and electronic effect of the chloride situated next to the other four-membered $\mathrm{C}-\mathrm{N}$ bond of $\mathbf{1 0}{ }^{7}$ 


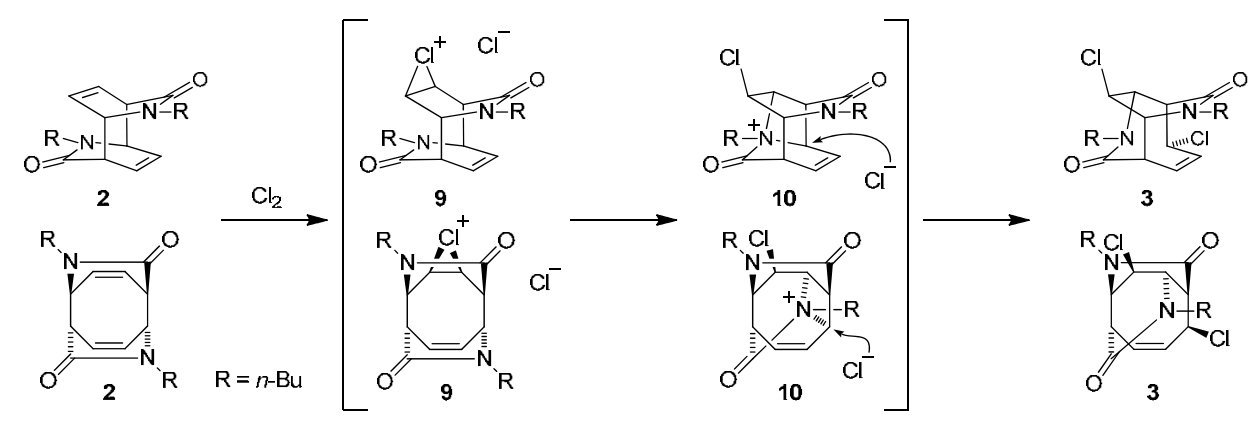

Scheme 3. Chlorination of 2 involves trapping of the initial chloronium ion by the amide.

Displacement of the allylic chloride in 3 by ethoxide (and other softer nucleophiles ${ }^{7}$ ) is believed to involve a double displacement. An $\mathrm{S}_{\mathrm{N}} 2$-compatible approach by an external nucleophile to the back- side of either $\mathrm{C}-\mathrm{Cl}$ bond of $\mathbf{3}$ is blocked by the tricyclic structure (see Scheme 3) and therefore the chlorides are immune to direct displacement. The amide nitrogen, however, is perfectly aligned to displace the allylic chloride, and does so even though this reforms the strained and reactive azetidinium ion 10. Once intermediate $\mathbf{1 0}$ is formed, an added nucleophile can replace the starting chloride in $\mathbf{3}$, and in this particular case yields $\mathbf{6}$. Additional evidence for the intermediacy of $\mathbf{1 0}$ in reactions of $\mathbf{3}$ includes the addition of an allyl Grignard reagent. Instead of alkylation by displacement of the nitrogen, the Grignard reagent attacks the carbonyl to give the tertiary alcohol $\mathbf{1 1} .^{7}$

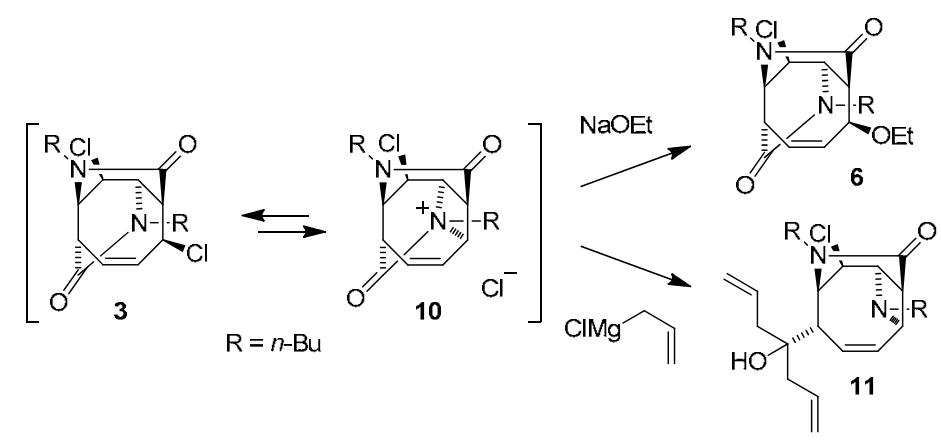

Scheme 4. Nucleophilic attack on dichloride 3.

\section{Results and Discussion}

With a goal of unwrapping these structures to give a functionalized acyclic carbon chain, and with the ethoxy- substituted $\mathbf{6}$ in hand, we turned to oxidative cleavage of the alkene. To this end we treated 6 with an excess of ozone in methanol-dichloromethane, followed by reduction with triphenylphosphine (Scheme 5). The resulting product, presumably dialdehyde 12a, was then reduced with sodium borohydride. With two epimerizable aldehydes in 12a it was deemed 
prudent to reduce them and solidify their stereochemistry. The diol product 13a appeared to be a single compound but it proved difficult to purify chromatographically because its $R_{f}$ was nearly identical to triphenylphosphine oxide. In addition, this product decomposed on repeated chromatography.

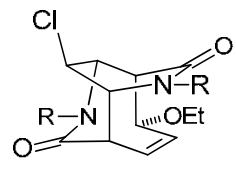

6

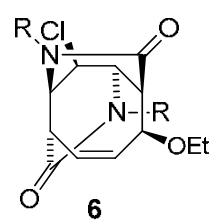

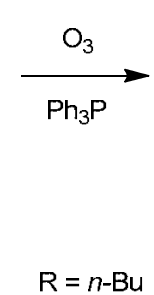
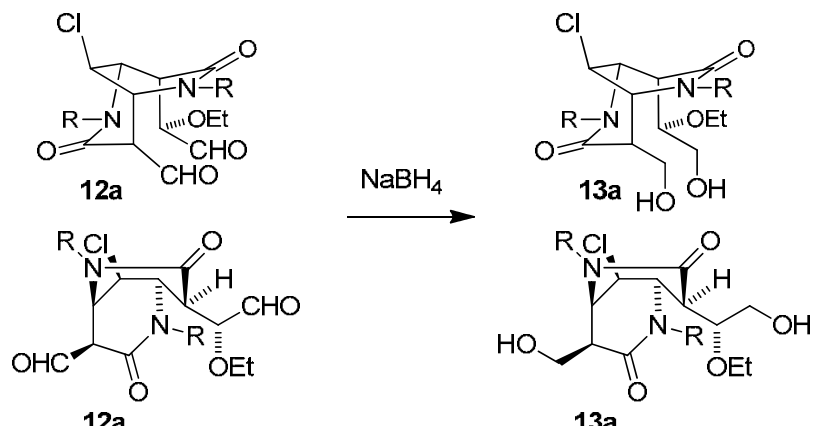

Scheme 5. Ozonolysis of the alkene.

As an alternative method, alkene cleavage was also studied, using sodium periodate and catalytic osmium tetroxide. While this reaction proceeded to give a single product, it was difficult to determine its precise structure. Confusingly, the presumed dialdehyde $\mathbf{1 2 b}$ had only one aldehyde absorbance in the ${ }^{13} \mathrm{C}$ - NMR spectrum, and a doublet at $4.8 \mathrm{ppm}$ in the ${ }^{1} \mathrm{H}$ - NMR spectrum suggested that the compound contained one hydrated aldehyde. ${ }^{10-12}$ But which compound $\mathbf{1 3}$ had the correct structure? Reduction with sodium borohydride gave a diol, $\mathbf{1 3 b}$. This product was different from the diol derived by ozonolysis.
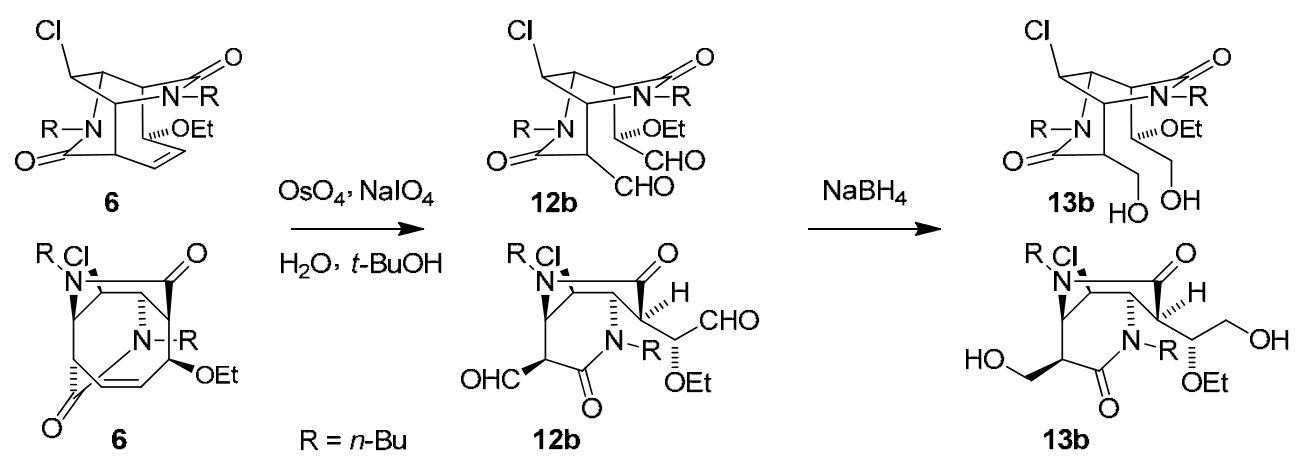

Scheme 6. Diol formation following alkene cleavage with periodate and osmium tetroxide gave a different diol $\mathbf{1 3 b}$.

Working with the reduced ozonolysis product, the difficultly separable mixture of 13a and triphenylphosphine oxide was used directly in the next step (Scheme 7). With anticipation of a potentially difficult double amide hydrolysis on the horizon, we chose to protect the alcohols of 13a as ethers. Notably, treatment of diol 13a with acetic anhydride or tosyl chloride gave the 
expected products, but when 13a treated with sodium hydride and benzyl bromide a complex mixture resulted. Nevertheless, when the diol was treated with a mixture of trimethyloxonium tetrafluoroborate and proton sponge, a dimethyl ether 14 was isolated in $60 \%$ yield. This was a crystalline compound and we were able to obtain a crystal structure. A stereo-view of this compound is shown in Figure 1.

$13 \mathbf{a}$
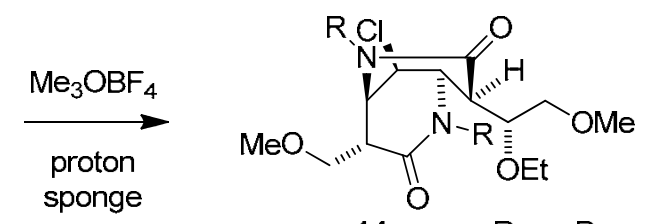

Scheme 7. Etherification of diol 13a.

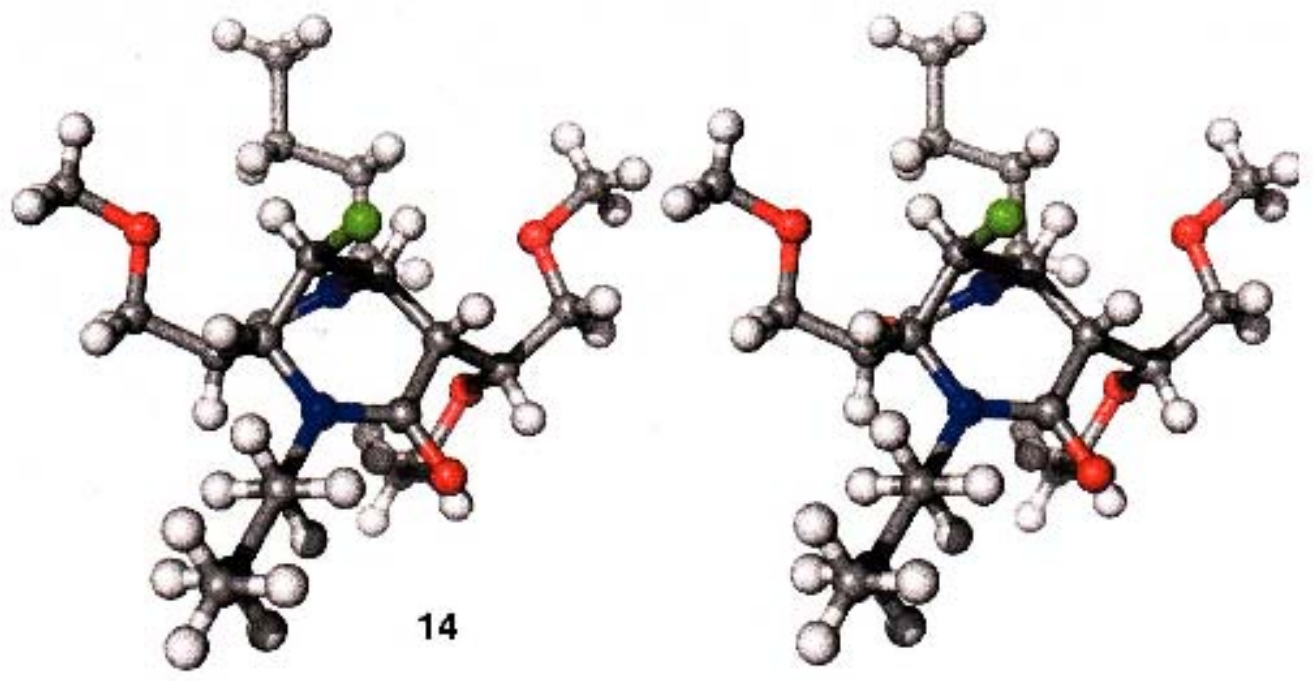

Figure 1. Stereo-image of the crystal structure of the dimethyl ether 14.

With some surprise, the crystal structure of di-ether 14 showed unexpected stereochemistry at one center. Undoubtedly this resulted from epimerization of an aldehyde before hydride reduction (Scheme 8). The presence of the beta-dicarbonyl substructure in 12a would be expected to make that stereogenic center particularly labile. 


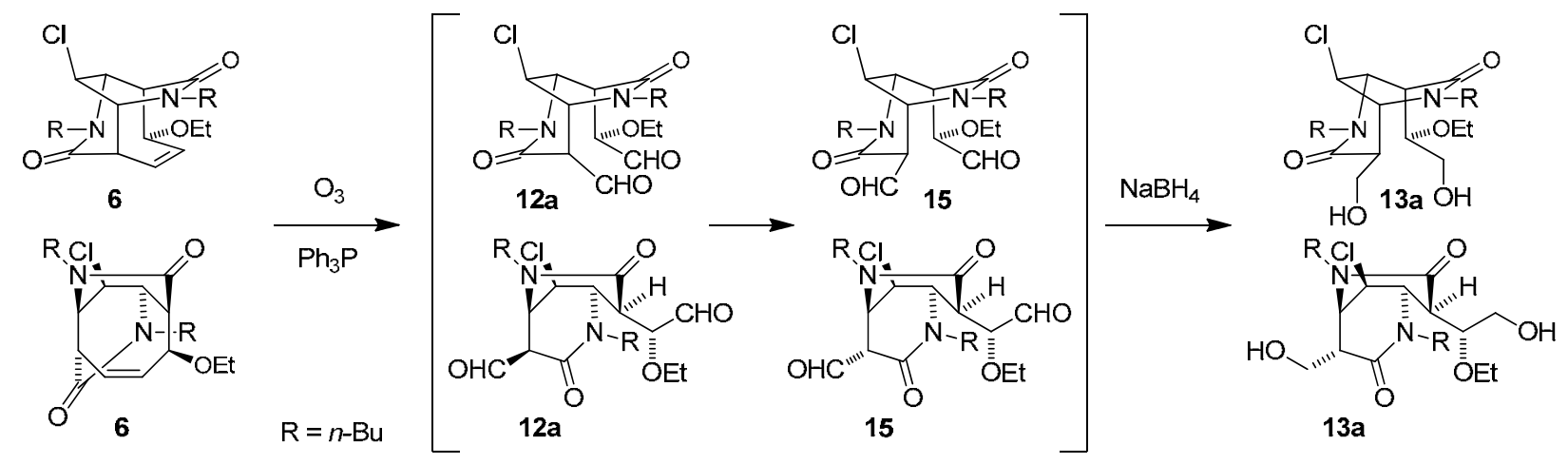

Scheme 8 (a correction of Scheme 5). Epimerization of one aldehyde occurs during ozonolysis.

Considering the structure of 12a, Scheme 9, the initially formed dialdehyde 12a has both aldehyde functional groups attached to the concave face of the [3.3.1] bicyclic system. One of the aldehydes is part of a beta-dicarbonyl and can therefore enolize to give 16. Tautomerization of this enol to $\mathbf{1 5}$ places the aldehyde on the less hindered exo face.
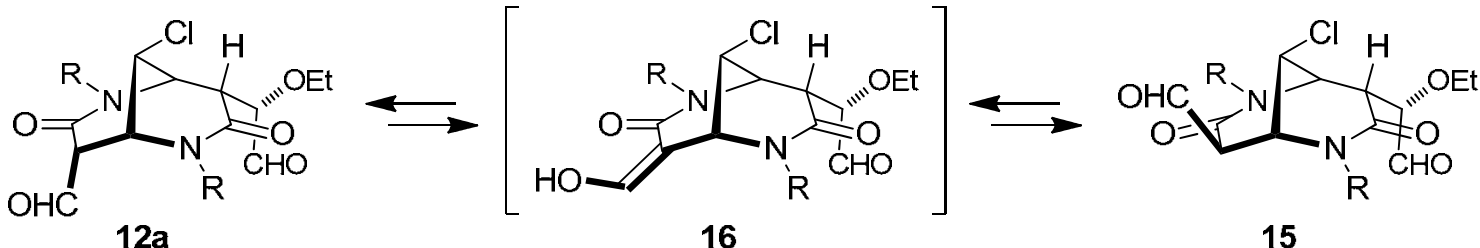

15

Scheme 9. Epimerization favors 15 over 12a.

The last step in the proposed sequence was ring-opening of the two tertiary amides (see Scheme 10). This transformation, regrettably, has not yet met the correct conditions. For example, treatment of $\mathbf{1 4}$ with $6 M$ hydrochloric acid at reflux for $3 \mathrm{~h}$ did not lead to any change, but concentrated $\mathrm{HCl}$ under similar conditions resulted in a complex mixture. Similarly, an excess of lithium tri-ethylborohydride gave a mixture of products that eluded characterization.
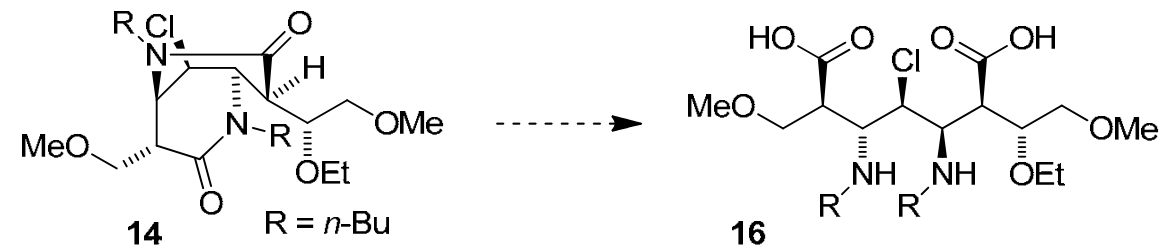

Scheme 10. Attempted ring opening of 14. 


\section{Conclusions}

Overall we have demonstrated (Scheme 11), that the tricyclic, achiral 2-pyridone photo-dimer 2 can be transformed into a complex bicyclic structure with six stereogenic centers. The transphotodimer $\mathbf{2}$ has a center of symmetry, however the rearrangement occurring with chlorination yields a chiral molecule with six stereogenic centers. Enantioselective halogenation would allow for asymmetric creation of products such as $\mathbf{1 0}$ and 14. In the current work, we have demonstrated that these unique cyclo-octane intermediates can be evolved into a ten-carbon product rich with functionality.

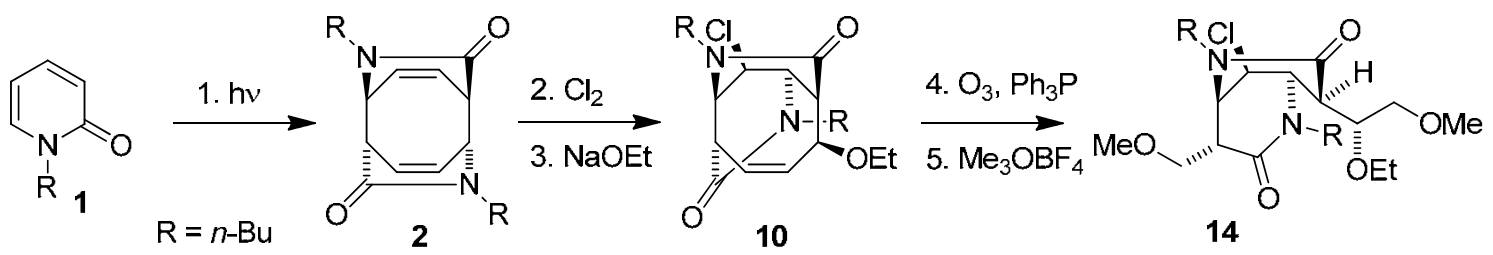

Scheme 11. A five-step sequence to bicyclic 14 from pyridone 1.

\section{Experimental Section}

General. All reactions were carried out under a nitrogen atmosphere unless otherwise noted. Dichloromethane was dried using an alumina column under argon (Glass Contour). NMR spectra were acquired in $\mathrm{CDCl}_{3}$ solvent at $400 \mathrm{MHz}\left({ }^{1} \mathrm{H}\right)$ and $100 \mathrm{MHz}\left({ }^{13} \mathrm{C}\right)$.

\section{$\left(1 R^{*}, 4 R^{*}, 5 R^{*}, 8 S^{*}, 9 R^{*}\right)$-2,6-Dibutyl-9-chloro-4-[1( $\left.S^{*}\right)$-ethoxy-2-hydroxyethyl]-8-}

hydroxymethyl-2,6-diazabicyclo[3.3.1]nonane-3,7-dione 13a. Into a $-78{ }^{\circ} \mathrm{C}$ solution of 6 (50 $\mathrm{mg}, 0.13 \mathrm{mmol})$ and sodium bicarbonate $(12 \mathrm{mg}, 0.14 \mathrm{mmol})$ in a mixture of dichloromethane (1 $\mathrm{mL})$ and methanol $(4 \mathrm{~mL})$ was bubbled a stream of ozone until a blue color appeared. The ozone addition tube was replaced with an argon tube and argon was added until the blue color had disappeared. To this solution was then added triphenylphosphine (69 $\mathrm{mg}, 0.26 \mathrm{mmol})$ and the resulting mixture was warmed to RT and stirred for $0.5 \mathrm{~h}$. After cooling to $0{ }^{\circ} \mathrm{C}$, sodium borohydride (44 mg, $1.2 \mathrm{mmol}$ ) was added and the mixture was warmed to RT and stirred for 1 h. After dilution with water, the aqueous phase was extracted with ethyl acetate and the combined organics were washed with saturated $\mathrm{NaCl}$ solution, dried over sodium sulfate, and filtered. Concentration in vacuo gave the crude product. Attempts to purify this mixture by silica gel chromatography resulted in decomposition of 13a. ${ }^{1} \mathrm{H}$ NMR $\left(\mathrm{CDCl}_{3}\right) \delta 4.75(\mathrm{~m}, 1 \mathrm{H}), 4.10$ $(\mathrm{m}, 2 \mathrm{H}), 3.94-3.60(\mathrm{~m}, 8 \mathrm{H}), 3.35(\mathrm{~m}, 1 \mathrm{H}), 3.23(\mathrm{~m}, 1 \mathrm{H}), 2.75(\mathrm{~m}, 2 \mathrm{H}), 2.58(\mathrm{~m}, 1 \mathrm{H}), 1.57 \sim 1.20$ (m, 8H), 1.15 (t, $J=7.0 \mathrm{~Hz}, 3 \mathrm{H}), 0.84(\mathrm{~m}, 6 \mathrm{H})$. 
$\left(1 R^{*}, 4 R^{*}, 5 R^{*}, 8 S^{*}, 9 R^{*}\right)-2,6-D i b u t y l-9-c h l o r 0-4-\left[1\left(S^{*}\right)\right.$-ethoxy-2-methoxyethyl]-8methoxymethyl-2,6-diazabicyclo[3.3.1]nonane-3,7-dione 14. To a mixture of 13a and triphenylphosphine oxide (1.32 g, containing $400 \mathrm{mg}, 1.1 \mathrm{mmol}$ of 13a) in dichloromethane ambient temperature was added trimethyloxonium tetrafluoroborate $(855 \mathrm{mg}, 5.8 \mathrm{mmol})$ and 1,8bis(dimethylamino)naphthalene $(823 \mathrm{mg}, 3.8 \mathrm{mmol})$, and the reaction was stirred for $6 \mathrm{~h}$. Following dilution with water, the aqueous phase was extracted with ethyl acetate. The combined extracts were concentrated until a solid precipitated. The mixture was filtered through Celite and the filtrate was concentrated. Flash chromatography $(2: 1$ hexanes/ethyl acetate) gave 14 as a light yellow solid. mp $67{ }^{\circ} \mathrm{C} ;{ }^{1} \mathrm{H}$ - NMR $\left(\mathrm{CDCl}_{3}\right) \delta 4.76(\mathrm{t}, \mathrm{J}=4.0,1 \mathrm{H}), 4.35(\mathrm{q}, \mathrm{J}=5.0$, $1 \mathrm{H}), 4.05(\mathrm{~m}, 3 \mathrm{H}), 4.73(\mathrm{~m}, 4 \mathrm{H}), 3.57(\mathrm{~m}, 2 \mathrm{H}), 3.35(\mathrm{~s}, 3 \mathrm{H}), 3.32(\mathrm{~s}, 3 \mathrm{H}), 3.35 \sim 3.25(\mathrm{~m}, 2 \mathrm{H})$, $3.00(\mathrm{~m}, 1 \mathrm{H}), 2.74(\mathrm{~m}, 1 \mathrm{H}), 2.50(\mathrm{~m}, 1 \mathrm{H}), 1.60 \sim 1.20(\mathrm{~m}, 8 \mathrm{H}), 1.10(\mathrm{t}, \mathrm{J}=7.0,3 \mathrm{H}), 0.89(\mathrm{t}, \mathrm{J}=$ 7.5, 6H); ${ }^{13} \mathrm{C} \mathrm{NMR}\left(\mathrm{CDCl}_{3}\right) \delta 167.0,166.8,74.9,74.0,73.5,65.1,60.8,59.5,59.4,56.5,53.3$, 49.6, 48.3, 45.8, 43.5, 29.8, 29.4, 20.5, 20.4, 15.7, 14.3, 14.1. Exact mass: Calcd. for $\mathrm{C}_{22} \mathrm{H}_{39} \mathrm{~N}_{2} \mathrm{O}_{5} \mathrm{Cl}: 469.2445(\mathrm{M}[35]+\mathrm{Na})$; found: 469.2441 .

\section{Acknowledgments}

This work was supported by grants from the National Institutes of Health and from Temple University.

\section{References}

1. Sieburth, S. McN.; Cunard, N. T. Tetrahedron 1996, 52, 6251.

2. Sieburth, S. McN., In Advances in Cycloaddition, Harmata, M., Eds.; JAI: Greenwich, CT. Vol. 5, 1999, 85-118.

3. Sieburth, S. McN., In CRC Handbook of Organic Photochemistry and Photobiology, Horspool, W., Lenci, F., Eds.; CRC Press: Boca Raton, FL. 2004, 103/1-103/18.

4. McGee, K. F. J.; Al-Tel, T. H.; Sieburth, S. M. Synthesis 2001, 8, 1185.

5. Lim, Y.; Li, T.; Chen, P.; Schreiber, P.; Kutnetsova, L.; Carroll, P. J.; Lauher, J. W.; Sieburth, S. McN. Org. Lett. 2005, 7, 5413.

6. Ader, T. A.; Champey, C. A.; Kuznetsova, L. V.; Li, T.; Lim, Y.-H.; Rucando, D.; Sieburth, S. McN. Org. Lett. 2001, 3, 2165.

7. Chen, P.; Carroll, P. J.; Sieburth, S. McN. Synthesis 2007, 2351.

8. Hageman, H. A. Org. React. 1953, 7, 198.

9. Cooley, J. H.; Evain, E. J. Synthesis 1989, 1.

10. Kamiya, T.; Saito, Y.; Hashimoto, M.; Seki, H. Tetrahedron 1972, 28, 899.

11. Borch, R. F.; Hoye, T. R.; Swanson, T. A. J. Med. Chem. 1984, 27, 490.

12. Breton, T.; Bashiardes, G.; Léger, J. M.; Kokoh, K. B. Eur. J. Org. Chem. 2007, 1567. 